\title{
Les universités face aux enjeux de la formation interdisciplinaire
}

Il existe depuis longtemps, au niveau de l'enseignement supérieur, des cursus qui ont un caractère pluridisciplinaire, voire interdisciplinaire. L'exemple des grandes écoles en montre même la banalité ; la diversité aussi, chacune des multiples sciences de l'ingénieur couvrant l'éventail des disciplines correspondant à ses objectifs propres de formation. Cette diversité illustre d'ailleurs une des grandes caractéristiques de ces assemblages : le pragmatisme. Mais, contrairement à une idée couramment admise, il en va de même dans les universités. Une analyse approfondie des formations anciennement dites « de $3^{\mathrm{e}}$ cycle » montrerait que le caractère disciplinaire de leurs intitulés n'est souvent qu'une commodité institutionnelle confortant une routine intellectuelle. Tout au plus peut-on penser que le spectre des disciplines associées dans leur contenu est moins ouvert que dans les enseignements des grandes écoles. Là encore, c'est l'objectif de la formation qui commande.

Bien que l'étant, aucune de ces formations ne se déclare interdisciplinaire : les grandes écoles forment chacune à un des grands "corps de l'État», les universités incarnent et ont en charge de reproduire le découpage des sciences, fort justement dit, précisément, «universitaire».

Le fait nouveau - il serait intéressant de bien le dater - est que des formations qui se déclarent, elles, interdisciplinaires apparaissent dans les universités. Parfois, d'ailleurs, en association avec les grandes écoles. Un rapide tour d'horizon fait récemment (à l'occasion des Journées 2007 de l'association Natures Sciences Sociétés Dialogues, intitulées «Les formations interdisciplinaires: expériences, problèmes, perspectives ») révèle qu'elles sont même fort nombreuses. Il faut sans doute attribuer ce foisonnement $\mathrm{d}$ 'initiatives au souci des universités de développer des formations à caractère professionnel; il s'agit de «masters » et, souvent, plus particulièrement de «masters pro». Mais ceci pourrait se faire sans qu'en soit nécessairement souligné le caractère interdisciplinaire. Le fait qu'il le soit a apparemment son importance : c'est une façon de souligner l'originalité de la formation proposée. On remarquera que cela se fait en prenant, en quelque sorte, le soin de se démarquer de l'image disciplinaire universitaire.

Ainsi, les universités viennent se placer sur le terrain des grandes écoles et, plus généralement, de toutes les écoles qui forment des ingénieurs et des cadres supérieurs de tous ordres. Ce faisant, elles découvrent combien leurs structures institutionnelles et leur organisation des enseignements, les habitudes intellectuelles et corporatives qui président à leur fonctionnement et les rapports sociaux - en particulier de pouvoir - que celles-ci induisent deviennent des tares comparativement à celles et ceux des écoles qu'elles ont à concurrencer. Ceci est abondamment ressorti des débats des Journées qui viennent d'être évoquées. Le risque qui en résulte est que l'interdisciplinarité revendiquée dans l'intitulé des masters en question ne soit plus proche d'un affichage de marketing que d'une réalité scientifique. Mais, du moins, le grand intérêt de ce volontarisme, même s'il peut être parfois plus subi que choisi, est qu'il lance une dynamique à laquelle il sera de plus en plus difficile d'échapper. C'est le grand mérite de celles et ceux qui prennent les initiatives et en assument les tâches et les difficultés que d'ouvrir, parfois avec un enthousiasme rapidement mis à rude épreuve, ce chantier nouveau pour les universités. La question est posée de savoir si celles-ci pourront relever le défi auquel elles ont à faire face. C'est en premier lieu une question de moyens, car l'interdisciplinarité ajoute ses coûts propres à ceux de l'enseignement habituel. Et ces coûts sont élevés, si l'on veut réellement en prendre les exigences en charge. Mais c'est aussi un défi sur le plan intellectuel qui leur est jeté.

De ce point de vue, les expériences de masters interdisciplinaires qui ont été présentées et discutées au cours des Journées de Nature Sciences Sociétés Dialogues ont été riches d'enseignements. Elles ont montré l'extrême diversité des cas de figure. Celle-ci est, certes, d'abord 
fonction des objectifs poursuivis et des contextes dans lesquels les créations se situent. Et de ce point de vue, la gamme va de pluridisciplinarités conçues en vue de donner à de futurs praticiens des compétences multiples et complémentaires à des interdisciplinarités se construisant pour couvrir des champs de recherche nouveaux, étendus et complexes. En outre, dans un cas comme dans l'autre, on peut avoir affaire à des pluridisciplinarités ou à des interdisciplinarités de proximité ou élargies. On retrouve là le pragmatisme découlant des objectifs de formation poursuivis, évoqué ci-dessus. Mais intervient aussi, et de façon d'une certaine manière prépondérante, la personnalité des animateurs, et notamment le degré de leur adhésion à une perspective réellement interdisciplinaire, ainsi que la conception qu'ils se font de celle-ci. Ceci se traduit dans les procédures pédagogiques mises en œuvre, dans la place qu'elles accordent à la confrontation des disciplines, bien sûr, mais aussi - trait moins évident - dans le rôle qu'elles donnent aux étudiants dans leur propre formation et notamment dans la place qu'elles font à leur initiative et à leur réflexivité, individuelles et collectives, en jouant sur la diversité de leurs formations initiales. Car un des problèmes majeurs de ces masters interdisciplinaires - même si la situation de ce point de vue n'est évidemment pas la même selon que l'on est dans une configuration de pluridisciplinarité ou d'interdisciplinarité de proximité ou élargie - est de trouver des formes d'enseignement répondant à l'hétérogénéité des publics d'étudiants.

Il serait très regrettable que cette richesse se perde dans son foisonnement. Pour qu'elle devienne une ressource collective, il faut pouvoir la partager. Tel était déjà le but des Journées dont il a été question tout au long de ces lignes. Il reste à en rendre compte de façon aussi complète, claire et synthétique que possible, pour qu'elle serve à la réflexion collective et inspire les expériences en cours ou à venir. C'est ce à quoi nous allons maintenant nous employer.

Marcel Jollivet

To access this journal online: www.edpsciences.org 